\title{
The Dark Side of the Propagators: analytical approach to QCD in the infrared of Minkowski space
}

\author{
Fabio SIRINGO* \\ Dipartimento di Fisica e Astronomia, Università di Catania \\ E-mail: Eabio.siringodct.infn.it
}

\begin{abstract}
Analytical functions for the propagators of QCD, including a set of chiral quarks, are derived by a one-loop massive expansion in the Landau gauge, and are studied in Minkowski space, yielding a direct proof of positivity violation and confinement from first principles. Universal scaling properties are predicted for the inverse dressing functions and shown to be satisfied by the lattice data. Complex conjugated poles are found for the gluon propagator.
\end{abstract}

34th annual International Symposium on Lattice Field Theory

24-30 July 2016

University of Southampton, UK

\footnotetext{
*Speaker.
} 


\section{Introduction}

Most of the non-perturbative approaches to QCD rely on numerical calculations in the Euclidean space, where synergic studies by lattice simulations, Schwinger-Dyson equations and, more recently, by variational methods [1-3] have drawn a clear picture for the propagators deep in the IR. However, many important dynamical information cannot be extracted by the Euclidean formalism, unless we have an analytic function that can be continued to the physical space or the whole numerical analysis is carried out in Minkowski space [4]. Thus, it is still discussed if the propagators have poles, while some evidence of positivity violation has been demonstrated by indirect arguments. By a linear regularization strategy, a Källen-Lehmann spectral function was reconstructed in Ref.[5] from the lattice data of the gluon propagator, giving some direct evidence for positivity violation and the absence of any discrete mass pole on the physical real axis.

Quite recently, an analytical approach has been proposed that is based on a different expansion point for the exact Lagrangian of pure Yang-Mills theory in the Landau gauge [6,7]. The new expansion is around a massive free-particle propagator, yielding a massive loop expansion with massive particles in the internal lines of the Feynman graphs. From first principles, without adding spurious counterterms or phenomenological parameters, at one-loop the expansion provides analytical universal functions for the dressing functions, predicting some scaling properties that are satisfied by the data of lattice simulations. In the Euclidean space and Landau gauge, the massive expansion is in impressive agreement with the lattice data and the one-loop propagators are analytic functions that can be easily continued and studied in Minkowski space. In this paper, a concise review of the massive expansion [7] is presented, in its full version of Ref. [8], containing a set of chiral quarks. The method has the merit of providing an analytical and consistent framework for the study of QCD in the infrared and of disclosing the analytic properties of the propagators in Minkowski space. While based on a perturbative expansion, the method has a variational nature, with an accuracy that can be increased by tuning the mass ratio and the subtraction point to optimal values that minimize the effect of higher loops. In that sense, the method can be seen as a special case of the optimized perturbation theory that has been discussed by many authors in the past $[9,10]$ and has been recently improved by RG methods [11]. The study of the spectral functions gives direct predictions on the dynamics of quarks and gluons. For instance, no poles are found for the gluon on the physical positive real axis and the positivity constraints are strongly violated for quarks and gluons, as expected for confined degrees of freedom. Moreover, complex conjugated poles are found for the gluon propagator, confirming the i-particle scenario [12] predicted by the refined version [13-15] of the Gribov-Zwanziger model [16].

\section{The Massive Expansion in the Chiral Limit}

The total action of QCD, including $N_{f}$ massless chiral quarks, can be written as $\mathcal{S}_{\text {tot }}=\mathcal{S}_{0}+\mathcal{S}_{I}$, where the free-particle term $\mathcal{S}_{0}$ is the usual quadratic part in terms of the standard free-particle propagators of gluons, quarks and ghosts, namely $\Delta_{0}, S_{0}$ and $\mathcal{G}_{0}$, respectively. As shown in Refs. [7,8] we may add and subtract the arbitrary terms $\delta \mathcal{S}_{g}, \delta \mathcal{S}_{q}$ in the total action

$$
\mathcal{S}_{0} \rightarrow \mathcal{S}_{0}+\delta \mathcal{S}_{q}+\delta \mathcal{S}_{g}, \quad \mathcal{S}_{I} \rightarrow \mathcal{S}_{I}-\delta \mathcal{S}_{q}-\delta \mathcal{S}_{g}
$$


and take

$$
\delta \mathcal{S}_{g}=\frac{1}{2} \int A_{a \mu}(x) \delta_{a b} \delta \Gamma_{g}^{\mu v}(x, y) A_{b v}(y) \mathrm{d}^{d} x \mathrm{~d}^{d} y, \quad \delta \mathcal{S}_{q}=\sum_{i=1}^{N_{f}} \int \bar{\Psi}_{i}(x) \delta \Gamma_{q}(x, y) \Psi_{i}(y) \mathrm{d}^{d} x \mathrm{~d}^{d} y
$$

where the vertex functions $\delta \Gamma_{g}, \delta \Gamma_{q}$ are given by a shift of the inverse propagators

$$
\delta \Gamma_{g}^{\mu v}(x, y)=\left[\Delta_{m}^{-1^{\mu v}}(x, y)-\Delta_{0}^{-1}{ }^{\mu v}(x, y)\right], \quad \delta \Gamma_{q}(x, y)=\left[S_{M}^{-1}(x, y)-S_{0}^{-1}(x, y)\right]
$$

and $\Delta_{m}{ }^{\mu v}, S_{M}$ are massive free-particle propagators

$$
\begin{aligned}
\Delta_{m}^{-1}{ }^{\mu v}(p) & =\Delta_{m}(p)^{-1} t^{\mu v}(p)+\frac{-p^{2}}{\xi} \ell^{\mu v}(p) \\
\Delta_{m}(p)^{-1} & =-p^{2}+m^{2}, \quad S_{M}(p)^{-1}=\not p-M .
\end{aligned}
$$

Here $t^{\mu v}, l^{\mu v}$ are Lorentz projectors and the masses $m$ and $M$ are totally arbitrary. Since $\delta \mathcal{S}_{q}$ and $\delta \mathcal{S}_{g}$ are added and subtracted again, the total action cannot depend on the masses, but any expansion in powers of the new shifted interaction $\mathcal{S}_{I} \rightarrow \mathcal{S}_{I}-\delta \mathcal{S}_{q}-\delta \mathcal{S}_{g}$ is going to depend on them at any finite order because of the truncation. Thus, while we are not changing the content of the theory, the emerging perturbative approximation is going to depend on the masses and can be optimized by a choice of $m$ and $M$ that minimizes the effects of higher orders, yielding a variational tool disguised to look like a perturbative method [7,8]. The idea is not new and goes back to the works on the Gaussian effective potential [9,17-25] where an unknown mass parameter was inserted in the zeroth order propagator and subtracted from the interaction, yielding a pure variational approximation with the mass that acts as a variational parameter.

The shifts $\delta S_{q}, \delta S_{g}$ have two effects on the resulting perturbative expansion: the free-particle propagators are replaced by massive propagators and new two-point vertices are added to the interaction, arising from the counterterms $\delta \Gamma_{g}=m^{2}$ and $\delta \Gamma_{q}(p)=-M$. We work in Landau gauge, the optimal choice for the massive expansion [7], the gluon propagator is transverse and we can drop projectors and all color indices. We can use the standard formalism of Feynman graphs with massive zeroth order propagators $\Delta_{m}, S_{M}$. Assuming that the effective coupling never reaches values that are too large [7], we may neglect higher loops and take a double expansion in powers of the total interaction and in powers of the coupling, retaining graphs with $n$ vertices at most and no more than $\ell$ loops. The graphs contributing to the quark and ghost self-energy and to the gluon polarization are shown in Fig.W up to the third order and one-loop.

\section{Pure Yang-Mills theory}

The dressed propagators of pure SU(N) Yang-Mills theory can be written as

$$
\Delta(p)^{-1}=-p^{2}+\frac{5}{8} \alpha m^{2}-[\Pi(p)-\Pi(0)], \quad \mathcal{G}(p)^{-1}=p^{2}-\Sigma_{g h}(p)
$$

where the ghost self-energy $\Sigma_{g h}$ and the gluon polarization $\Pi$ were evaluated in Ref. [7] as a sum of the graphs in Fig.W (omitting quark loops) and $\alpha$ is an effective coupling. The one-loop gluon and ghost propagators are made finite by standard wave function renormalization and explicit analytic expressions were derived by dimensional regularization in Ref. [7]. 
It is useful to introduce the adimensional ghost and gluon dressing functions

$$
\chi(p)=p^{2} \mathcal{G}(p), \quad J(p)=-p^{2} \Delta(p) .
$$

They can be written as

$$
[\alpha \chi(s)]^{-1}=G(s)+G_{0} \quad[\alpha J(s)]^{-1}=F(s)+F_{0}
$$

where $s=-p^{2} / m^{2}$ is the Euclidean momentum and the two adimensional functions $F(s), G(s)$ are given by the polarization and self energy graphs in Fig.W, while all the constants are grouped together in the finite one-loop renormalization constants $F_{0}$ and $G_{0}$ that are the only free parameters to be optimized. Being equivalent to a variation of the subtraction point, any change of the additive constant can be seen as a variation of the renormalization scheme yielding a special case of optimized perturbation theory that has been proven to be very effective for the convergence of the expansion [10].

A very important consequence of Eq.(B.3) is that, up to an arbitrary multiplicative renormalization constant, the inverse dressing functions are given by the universal functions $F(s)$ and $G(s)$ up to an additive renormalization constant. Such scaling property is satisfied quite well by the lattice data for $\mathrm{SU}(2)$ and $\mathrm{SU}(3)$ that collapse on the same universal curves $F(s), G(s)$ in the infrared $[6-8,26]$ as shown in Fig.D. That scaling property confirms that higher order terms can be made negligible by an optimized choice of the constants $F_{0}, G_{0}$. Since ghosts are not coupled with quarks at one-loop, their scaling properties holds even for unquenched data in Fig.】.

The gluon propagator can be continued to Minkowski space by setting $s=-p^{2} / m^{2}-i \varepsilon$ and the resulting complex function is shown in Fig. [3. The imaginary part has a cut for $p^{2}>0$ where it defines a spectral function. The lack of any sharp peak or pole on the real axis and the violation of positivity can be regarded as a direct proof of confinement.

Out of the real axis, in the complex plane, the propagator has two conjugated poles at $p^{2} \approx$ $(0.16 \pm 0.60 i) \mathrm{GeV}^{2}$, close to the imaginary axis, as predicted by the $i$-particle scenario [12] emerging from the refined version [13-15] of the Gribov-Zwanziger model [16].

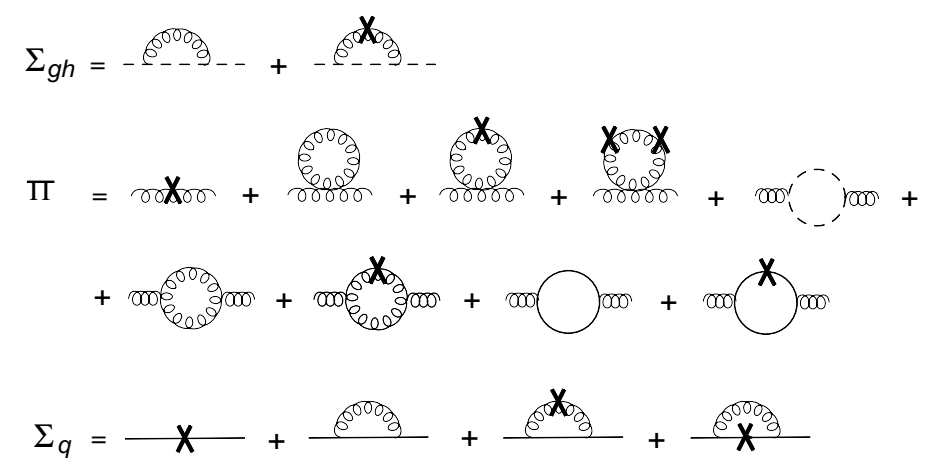

Figure 1: Two-point graphs with no more than three vertices and no more than one loop. The crosses are the counterterms $\delta \Gamma_{g}=m^{2}, \delta \Gamma_{q}=-M$. 


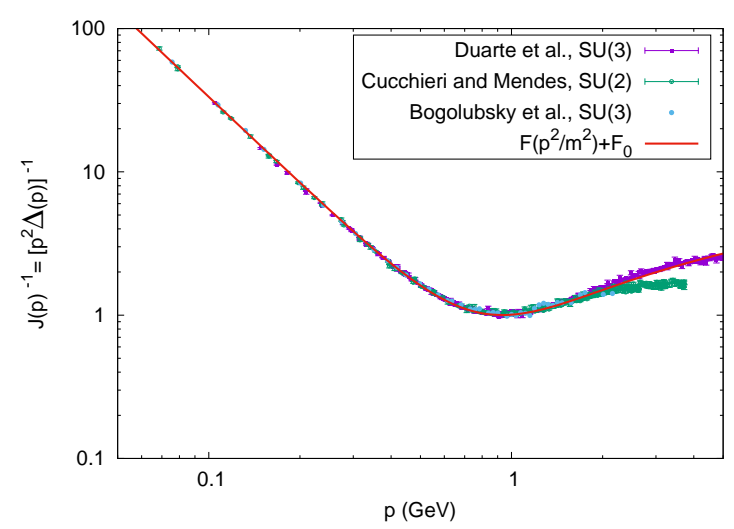

(a) Gluon

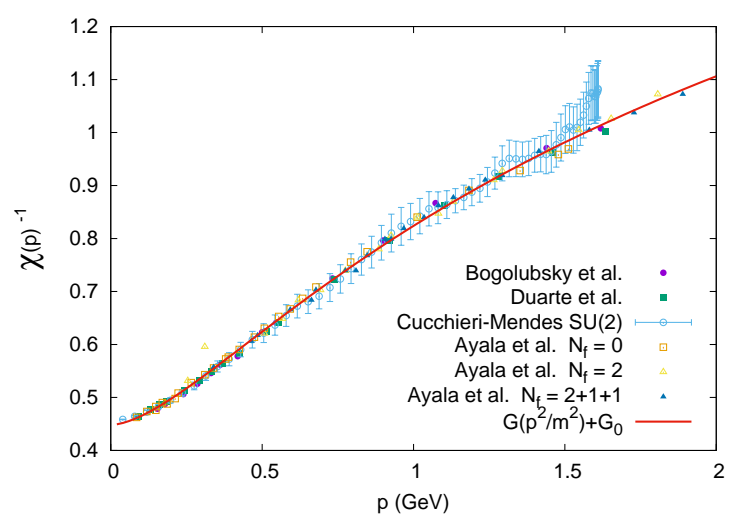

(b) Ghost

Figure 2: The inverse gluon and ghost dressing functions, $z J^{-1}(p / x)+y$ and $z^{\prime} \chi^{-1}(p / x)+y^{\prime}$, respectively, scaled by the parameters of Table 1. The lattice data of Bogolubsky et al.[27] and Duarte et al.[28] for $S U(3)$ are compared with the data of Cucchieri and Mendes [29,30] for $S U(2)$ and with the unquenched data of Ayala et al.[31] for full QCD with $N_{f}=2$ and $N_{f}=2+1+1$. The solid curves (red lines) are the one-loop universal functions $F(s), G(s)$ for $s=p^{2} / m^{2}, m=0.73$ $\mathrm{GeV}$ and shifted by the optimal constants $F_{0}=-1.05, G_{0}=0.24$.

\begin{tabular}{cccccccc}
\hline \hline Data set & $N$ & $N_{f}$ & $x$ & $y$ & $z$ & $y^{\prime}$ & $z^{\prime}$ \\
\hline Bogolubsky et al.[27] & 3 & 0 & 1 & 0 & 3.33 & 0 & 1.57 \\
Duarte et al.[28] & 3 & 0 & 1.1 & -0.146 & 2.65 & 0.097 & 1.08 \\
\hline Cucchieri-Mendes [29,30] & 2 & 0 & 0.858 & -0.254 & 1.69 & 0.196 & 1.09 \\
\hline Ayala et al.[31] & 3 & 0 & 0.933 & - & - & 0.045 & 1.17 \\
Ayala et al.[31] & 3 & 2 & 1.04 & - & - & 0.045 & 1.28 \\
Ayala et al.[31] & 3 & 4 & 1.04 & - & - & 0.045 & 1.28
\end{tabular}

Table 1: Scaling constants $x, y, z$ (gluon) and $y^{\prime}, z^{\prime}$ (ghost) used in Fig.】. The constant shifts $F_{0}=-1.05, G_{0}=0.24$ and the mass $m=0.73 \mathrm{GeV}$ are optimized by requiring that $x=1$ and $y=y^{\prime}=0$ for the lattice data of Bogolubsky et al.[27]

\section{Chiral QCD}

The inclusion of a set of chiral quarks requires the calculation of the quark loops contributing to the gluon polarization and the quark self-energy $\Sigma_{q}$. At one-loop, we must add all the graphs of Fig.⿴囗十.

As for pure Yang-Mills theory, the gluon dressing function is still given by Eq.(B.3) provided that a quark correction $\Delta F(s)$ is added to the function $F(s)$. Explicit results are derived in Ref. [8]. Besides being more rich on the real positive axis $p^{2}>0$, for $N_{f}=2$ the unquenched gluon propagator has more poles in the complex plane. For the optimal set $m=0.8 \mathrm{GeV}, M=0.65 \mathrm{GeV}$ We find two pairs of conjugated poles at $p^{2} \approx(1.69 \pm 0.1 i) \mathrm{GeV}^{2}$ and $p^{2} \approx(0.54 \pm 0.52 i) \mathrm{GeV}^{2}$.

The quark self energy $\Sigma_{q}$ is given by the tree term $\delta \Gamma_{q}=-M$ and three one-loop graphs, as 
GLUON PROPAGATOR - SU(3)

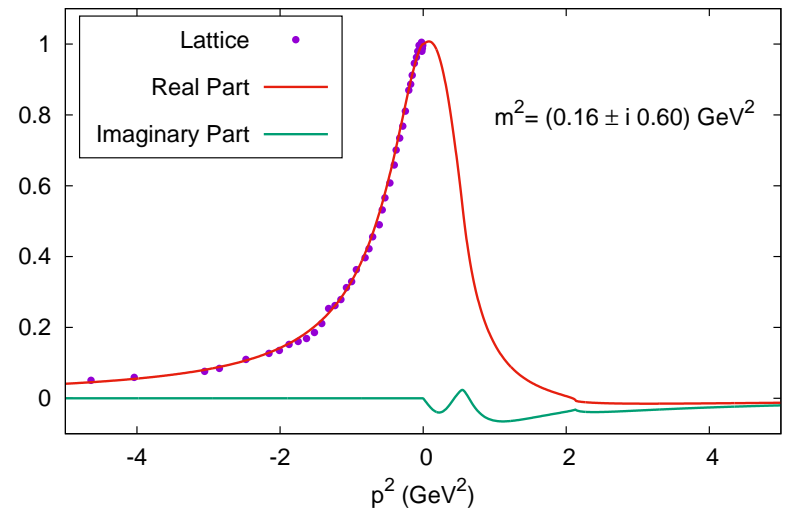

Figure 3: The real and the imaginary part of the gluon propagator of pure $S U(3)$ theory are displayed together with the lattice data of Ref.[27].

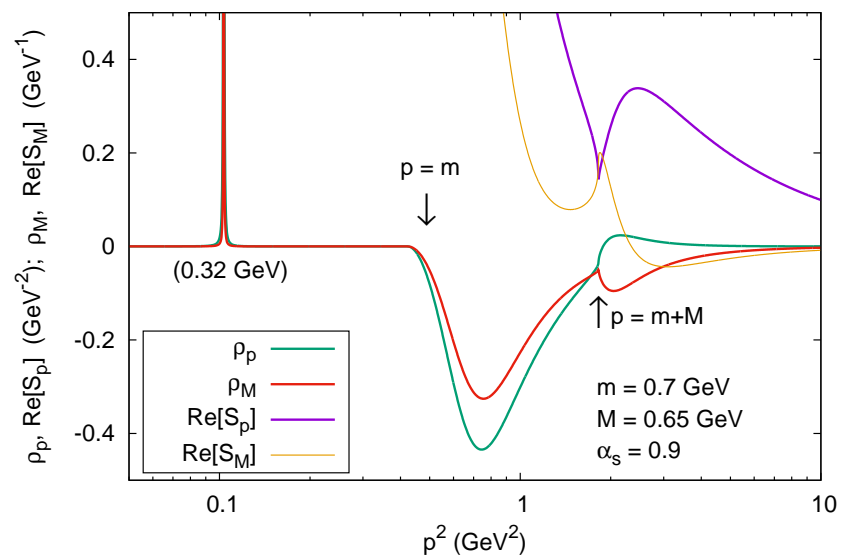

Figure 4: Details of the quark spectral functions for $\alpha_{s}=0.9, M=0.65 \mathrm{GeV}, m=0.7 \mathrm{GeV}$.

shown in Fig.W. The explicit analytical result can be continued and explored in Minkowski space as shown in Ref. [8]. The quark propagator can be written as

$$
S(p)=S_{p}\left(p^{2}\right) p+S_{M}\left(p^{2}\right)
$$

and the scalar functions $S_{p}, S_{m}$ can be continued to the complex plain. The imaginary parts have a cut on the real positive axis $p^{2}>0$ where we can define two spectral densities

$$
\rho_{M}\left(p^{2}\right)=-\frac{1}{\pi} \operatorname{Im} S_{M}\left(p^{2}\right), \quad \rho_{p}\left(p^{2}\right)=-\frac{1}{\pi} \operatorname{Im} S_{p}\left(p^{2}\right) .
$$

The spectral functions are shown in Fig.⿴囗十 for $\alpha_{s}=0.9, M=0.65 \mathrm{GeV}$. We recognize a discrete term in the spectral functions at the constituent quark mass $p \approx 0.32 \mathrm{GeV}$, that arises from the pole of the propagator. However the positivity conditions are strongly violated by the spectral functions, giving a direct proof of confinement. 


\section{References}

[1] F. Siringo and L. Marotta, Eur. Phys. J. C 44, 293 (2005).

[2] F. Siringo, Mod. Phys. Lett. A 29, 1450026 (2014), [arXiv:1308.4037].

[3] F. Siringo, Phys. Rev. D 88, 056020 (2013); Phys. Rev. D 89, 025005 (2014); Phys. Rev. D 90, 094021 (2014); Phys. Rev. D 92, 074034 (2015); arXiv:1507.05543.

[4] S. Strauss, C. S. Fischer, C. Kellermann, Phys. Rev. Lett. 109, 252001 (2012).

[5] D. Dudal, O. Oliveira, P. J. Silva, Phys. Rev. D 89, 014010 (2014).

[6] F. Siringo, arXiv:1509.05891.

[7] F. Siringo, Nucl. Phys. B 907, 572 (2016), [arXiv:1511.01015].

[8] F. Siringo, Phys. Rev. D 94, 114036 (2016), [arXiv:1605.07357].

[9] P.M. Stevenson, Phys. Rev. D 32, 1389 (1985).

[10] P.M. Stevenson, Nucl. Phys. B 868, 38 (2013); Nucl. Phys. B 910, 469 (2016).

[11] J.-L. Kneur and A. Neveu, Phys. Rev. D 81, 125012 (2010); Phys. Rev. D 85, 014005 (2012); Phys. Rev. D 88, 0704025 (2013); Phys. Rev. D 92, 074027 (2015).

[12] L. Baulieu, D. Dudal, M. S. Guimaraes, M. Q. Huber, S. P. Sorella, N. Vandersickel, D. Zwanziger, Phys.Rev.D 82, 025021 (2010).

[13] D. Dudal, J. A. Gracey, S. P. Sorella, N. Vandersickel, H. Verschelde, Phys. Rev. D 78, 065047 (2008).

[14] D.Dudal, S.P.Sorella, N.Vandersickel, H.Verschelde, Phys. Rev. D 77, 071501 (2008).

[15] D. Dudal, S. P. Sorella, N. Vandersickel, Phys. Rev. D 84, 065039 (2011).

[16] D. Zwanziger, Nucl. Phys. B 323, 513 (1989).

[17] F. Siringo, L. Marotta, Phys. Rev. D 78, 016003 (2008); Phys. Rev. D 74, 115001 (2006).

[18] F. Siringo, Phys. Rev. D 86, 076016 (2012), [arXiv: 1208.3592v2].

[19] I. Stancu and P. M. Stevenson, Phys. Rev. D 42, 2710 (1990).

[20] I. Stancu, Phys. Rev. D 43, 1283 (1991).

[21] M. Camarda, G.G.N. Angilella, R. Pucci, F. Siringo, Eur. Phys. J. B 33, 273 (2003).

[22] L. Marotta, M. Camarda, G.G.N. Angilella and F. Siringo, Phys. Rev. B 73, 104517 (2006).

[23] L. Marotta and F. Siringo, Mod. Phys. Lett. B, 26, 1250130 (2012).

[24] F. Siringo, Phys. Rev. D 62, 116009 (2000); Europhys. Lett. 59, 820 (2002).

[25] F. Siringo and L. Marotta, Int. J. Mod. Phys. A 25, 5865 (2010).

[26] F. Siringo, arXiv:1607.02040.

[27] I.L. Bogolubsky, E.M. Ilgenfritz, M. Muller-Preussker, A. Sternbeckc, Phys. Lett. B 676, 69 (2009).

[28] A. G. Duarte, O. Oliveira, P. J. Silva, Phys. Rev. D 94, 014502 (2016).

[29] A. Cucchieri and T. Mendes, Phys. Rev. D 78, 094503 (2008).

[30] A. Cucchieri and T. Mendes, Phys. Rev. Lett. 100, 241601 (2008).

[31] A. Ayala, A. Bashir, D. Binosi, M. Cristoforetti and J. Rodriguez-Quintero, Phys. Rev. D 86, 074512 (2012). 